\title{
Successful Distancing: Telemedicine in Gastroenterology and Hepatology During the COVID-19 Pandemic
}

\author{
Abhilash Perisetti ${ }^{1} \cdot$ Hemant Goyal $^{2,3}$ \\ Accepted: 19 January 2021 / Published online: 3 March 2021 \\ (c) The Author(s), under exclusive licence to Springer Science+Business Media, LLC part of Springer Nature 2021
}

\begin{abstract}
Telemedicine involves delivering healthcare and preventative care services to patients without the need for in-person encounters. Traditionally, telemedicine has been used for acute events (e.g., stroke, used to relay essential information to the emergency department) and chronic disease management (e.g., diabetes and chronic kidney disease management). Though the utilization of telemedicine in gastroenterology and hepatology has been modest at best, especially for inflammatory bowel diseases and chronic liver disease management, since the onset of coronavirus disease 2019 (COVID-19) pandemic, utilization of telemedicine in gastroenterology increased by $4000 \%$ in the first two weeks, equivalent to the last six years of growth before the pandemic. The Center for Medicare and Medicaid (CMS) relaxed rules for the use of telemedicine with easing restrictions on reimbursements, location, licensing requirements (across state lines), and the need for a prior provider-patient relationship. These changes increased the use of telemedicine in inpatient and outpatient settings for gastroenterology-related referrals. The use of inpatient telephonic or video consults helps provide timely care during the pandemic while conserving personal protective equipment and decreasing provider and patient exposure. Nevertheless, telehealth use comes at the cost of no direct patient contact and lesser reimbursements. The appropriate use of technology and equipment, training of healthcare providers, use of platforms that can be integrated into the electronic health record while protecting the privacy and the flow of information are essential components of telemedicine. Furthermore, encouraging patients to seek medical care remotely with the proper equipment and improving digital literacy without the need for physical examinations is a challenge, further compounded in elderly or hard-of-hearing patients and in patients who are more comfortable with in-person visits. The authors will systematically review and discuss how telemedicine can be integrated into the practice of gastroenterology and hepatology, with emphasis placed on discussing barriers to success and the ways they can be mitigated.
\end{abstract}

Keywords Telemedicine · Telehealth · COVID-19 · Gastroenterology · Hepatology · IBD · Pandemic · COVID-19 . Coronavirus $\cdot$ SARS-CoV-2

Hemant Goyal

doc.hemant@yahoo.com

Abhilash Perisetti

abhilash.perisetti@gmail.com

1 Department of Gastroenterology and Hepatology, University of Arkansas for Medical Sciences, Little Rock, AR, USA

2 The Wright Center for Graduate Medical Education, 501 S. Washington Avenue, Scranton, PA 18505, USA

3 Mercer University School of Medicine, 707 Pine St, Macon, GA 31201, USA

\section{Introduction}

Telemedicine is a term used to provide remote healthcare to patients without the need for in-person encounters [1]. Telehealth is a broader term that includes a wide range of methods used to communicate with patients using video portals and telephonic communication. The Health Resources and Services Administration (HRSA) of the US Department of Health and Human Services defines telehealth as "the use of electronic information, telecommunications technologies to support long-distance clinical healthcare, health-related education, public health, and administrative purposes" [2]. The technologies used vary widely but commonly include videoconferencing, storing or forwarding images, streaming media, landlines, and wireless communications [2]. Though 
telemedicine and telehealth are often used interchangeably, telemedicine is limited in its definition as "the delivery of healthcare services, where distance is a critical factor, by all healthcare professionals using technologies for the exchange of diagnosis, treatment, and prevention" [3]. Among these definitions, the decisive factor is "distance" without direct patient contact. Telemedicine includes multiple subtypes such as telemonitoring (patients wearing mobile devices and reporting to their providers about symptoms/disease progression), tele-education (webinars, interactive sessions provided to providers or patients or both), teleconsultation (remote ICU care, ED consultation), and telecare (video interaction to simulate a face-to-face visit in order to assist in diagnosis and treatment). Despite multiple barriers, telemedicine offers important advantages as outlined below (Table 1) [4].

Since its introduction, telemedicine has been extensively studied in both acute and chronic care settings. Teleconsultations are widely used in ED and ICU settings, where rapid access to patient information is essential and could be lifesaving [4]. One of the best examples includes acute stroke intervention provided by neurologists for patients admitted to remote hospitals [5]. Chronic disease management via telemedicine has gained significance in various fields such as cardiology, endocrinology, rheumatology, nephrology, and other areas where longitudinal metrics of patient data are essential for evaluating trends in quality measures (e.g., glycated hemoglobin, serum creatinine). Telemedicine has made inroads in the field of gastroenterology as well. Some of the most important areas within gastroenterology where telemedicine is utilized include inflammatory bowel disease
(IBD) [6, 7], GI motility [8], and HCV treatment monitoring [9]. The emergence of human infection with the severe acute respiratory syndrome coronavirus-2 (SARS-CoV-2) in December 2019 in Wuhan, China, and its rapid evolution into the coronavirus 2019 (COVID-19) pandemic in March 2020 significantly stressed healthcare systems [10]. Multiple organizations recommended several public health measures such as stay-at-home orders, social distancing, quarantines, and containment in the USA and globally [11-13]. These measures discouraged the face-to-face $(\mathrm{F} 2 \mathrm{~F})$ encounters that increased infection transmission risk.

Traditionally, the Center for Medicare and Medicaid Services (CMS) required the following criteria to be met to qualify for a telemedicine visit [14]: prior relationship (providers should have an established patient relationship with prior visits), location (patient should visit a nearby facility to access the remote visit), and modality (video conferencing technology should be available at the facility). Nonetheless, CMS expanded telemedicine benefits on an emergency and temporary basis (1135 waiver authority and Coronavirus Preparedness Response Supplemental Appropriations Act, March 27, 2020 [H.R.6074]) so as to remove the restriction of location (patients can receive care from home and at any facility) with CDC recommendations to limit in-person visits [15]. These changes, adopted by private insurers as well [16], were rapidly adopted by hospitals and healthcare institutions in order to incorporate telemedicine into GI practice in both outpatient and inpatient settings. Due to these changes, multiple studies were published in order to evaluate the efficacy, outcomes of telemedicine

Table 1 Advantages and barriers in telemedicine

Telemedicine

\begin{tabular}{ll}
\hline Advantages & Barriers
\end{tabular}

Access-Access to healthcare anywhere/anytime if internet connection is available

Legal - Coverage of malpractice differs across state lines and among insurance carriers

Financial-Low cost, flexible timings for both patients and providers

Delays-Could decrease backlogs and scheduling delays, especially during the pandemic

Stratification-Could stratify care with complex decision-making discussions requiring in-person visits

Preferences-Options to facilitate lifestyle preferences of the patients and avoid the commute

Monitoring-Frequent laboratory monitoring and patient education

Information sharing - Relay/ access of critical information. Option to have multiple providers from different specialties at one time

Communication-Discussion among PCP and specialists one platform, increased engagement of patients, increased practice efficiency, and reducing no-shows

Financial-Reimbursement issues and cost-sharing

Licensure-Requirement of licensures across multiple states and related paperwork

Education-Patient: Learning curve with software, need for an optimum user interface, training prior to use. Provider: Learning curve with navigating across the software

Equipment-Appropriate use of hardware and software, the related cost for patients and providers

Patient factors-Hearing/vision-related issues. Patient reliance on lip reading for communication, personal preference of in-person visits

Privacy concerns - Need for HIPPA compliance, privacy issues, risk, need for integration, risk of loss of information/hacking

Others_Loss of traditional "hands-on" physical examination, patientprovider relationship, lack of non-verbal gesture to communicate

Some of these barriers are dependent on state-specific licensure rules, insurance carriers. Providers/ancillary staff could engage in discussion with the patient prior to initiating a televisit. PCP—-primary care provider, HIPPA — health insurance portability, and accountability act 
in GI diseases extending well beyond IBD and HCV management. Yet, as the pandemic unfolds in different phases, uncertainty still exists about the most appropriate modality of telemedicine to use, the type of technology, the equipment needed, provider training, and audio-video compatibility. Furthermore, patient-related issues such as getting used to such technology, space availability, privacy concerns, and health insurance portability and accountability act (HIPPA)related issues exist. Given these, we aim to review the recent updates, including recently published literature on telemedicine and what could be expected in the future.

\section{Search Strategy}

A comprehensive search was performed using PubMed, Embase, Cochrane, OVID, Web of Science, ClinicalTrials. gov, Elsevier/Science Direct on December 6, 2020. Search terms used were-“gastroenterology," "gastrointestinal disease," "liver disease," "liver dysfunction," "liver transplantation," "inflammatory bowel disease," "Crohn's disease," "Ulcerative colitis," "IBD," "hepatitis C," "hepatitis C antigen," "telemedicine," "telehealth," "mobile health" or "videoconferencing." Initial search for telehealth or mobile health yielded 34,910 articles. A search using gastroenterology, liver disease, IBD, and hepatitis $C$ revealed 1,520,038 articles. After combining these two searches and using filters (human species and English language) and manual removal of duplicates, 302 published reports were finally reviewed in their full manuscripts.

\section{Role of Telemedicine in Gastroenterology During COVID-19}

Telemedicine through telephone and video has been used since 1960 in military and space where in-person visits were not feasible [17]. Increased availability of broadband technology increased the connectivity of different individuals from rural to urban areas [18]. Though telemedicine in gastroenterology has been explored only in chronic disease management of patients with IBD, HCV, and hepatology (Fig. 1) [19], COVID-19 provided a unique opportunity for healthcare institutions, gastroenterologists, and patients to adapt to online platforms to access healthcare in a safe environment in a timely manner. COVID-19 is rapidly changing and evolving with the emergence of extrapulmonary manifestations [20]. GI and liver symptoms have emerged as important manifestations of COVID-19, which can also precede respiratory symptoms, and in a few cases, can be the only symptoms [21-23]. Furthermore, taste changes among COVID-19 patients are observed in up to $50 \%$ of cases [24]. Hence, recognizing these symptoms early in the disease process can help prompt identification, isolation, quarantine, and management. Multiple studies reported a significant decrease in endoscopy volume during the pandemic due to widespread stay-at-home orders.

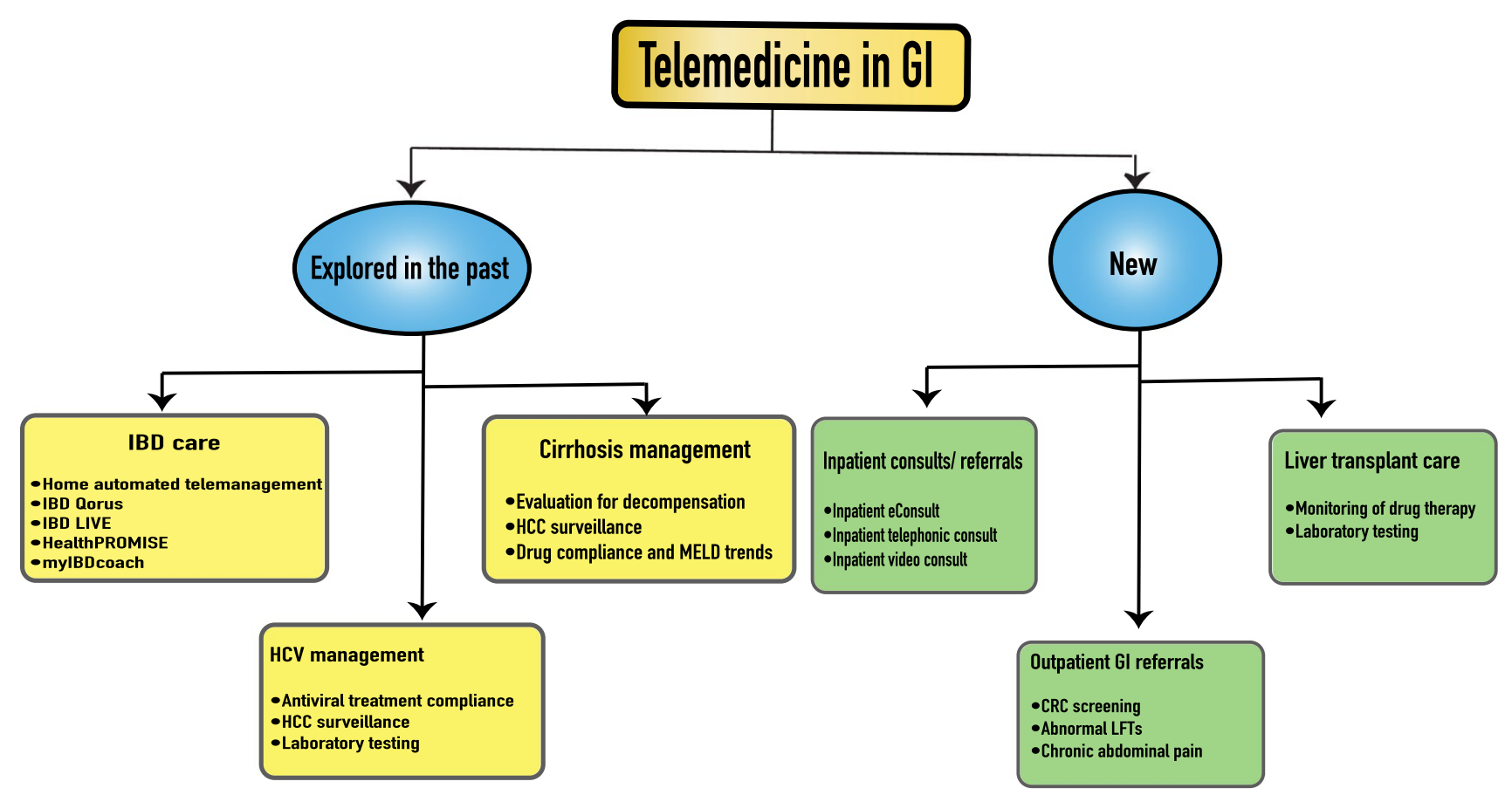

Fig. 1 Contributions of telemedicine to the practice of gastroenterology, past and the present 
Moreover, due to overwhelmed healthcare systems, endoscopy units were utilized as COVID-19 units [25], with consequent widespread delays and an increasing backlog of elective cases [26]. These changes forced providers to communicate with patients via telemedicine route.

Given this need, there was a pressing demand to increase the telemedicine options available during the pandemic, compounded by the rescheduling of elective cases and increased wait times. Fortunately, governing bodies in the USA (CMS), Asia, and Europe have eased the restrictions on using and increased reimbursement opportunities to further facilitate the use of telemedicine [15, 27]. Despite recent explosive growth, it is unclear which model to adapt, especially in outpatient GI practice in patients without a history of IBD or HCV. Further, uncertainty about the reimbursement platform, licensure needed, malpractice coverages, software, and equipment needed created a steep learning curve for the providers.

\section{Telemedicine Models and GI Disease Management}

Patients requiring consultation by a gastroenterologist need to be first triaged by the urgency of the consultation. Several factors should be considered, such as time sensitivity of the diagnosis, adverse effects of delay in diagnosis, and the need for additional evaluation prior to the consultation. Before establishing a telemedicine visit, adequate availability of hardware such as the presence of a secure phone line, a microphone, a video monitor, and related connections and HIPPA-compliant software with an Internet connection is needed [28]. Multiple platforms have been used to facilitate this process that could be either integrated into the electronic health record (EHR)—Epic ${ }^{\mathrm{TM}}$ (Epic Systems Corporation, Verona, WI) virtual platform, American Well ${ }^{\mathrm{TM}}$, GI OnDemand ${ }^{\circledR}$ (American College of Gastroenterology endorsed member benefit) or non-integrated platforms-Cisco WebEx meetings ${ }^{\mathrm{TM}}$, Doxy.me ${ }^{\mathrm{TM}}$, Doximity ${ }^{\mathrm{TM}}$, Vsee ${ }^{\mathrm{TM}}, \mathrm{Skype}^{\mathrm{TM}}$, GoToMeeting $^{\mathrm{TM}}$, and Zoom for Healthcare ${ }^{\mathrm{TM}}$ (Table 2) [29-34]. Recently, the US Department of Health eased video visit restrictions by using applications such as Apple FaceTime, WhatsApp, Facebook Messenger, and Google Hangouts, provided they are used in good faith [15]. Once appropriate hardware and software are available, screening of the referral should be made either by supporting staff or the GI provider based on the time sensitivity of the diagnosis.

Table 2 Common platforms that could be potentially utilized for providing GI telemedicine

\begin{tabular}{|c|c|}
\hline Name & Information \\
\hline Epic TM & Depending on the type of contract, it could be built into the existing Epic EHR \\
\hline American Well ${ }^{\mathrm{TM}}$ & Used by LiveHealth online, contracts Anthem ${ }^{\circledR} /$ Wellpoint ${ }^{\circledR}$ \\
\hline GI OnDemand ${ }^{\mathrm{TM}}$ & $\begin{array}{l}\text { An ACG-endorsed platform offers secure video, end-to-end practice management tools, } \\
\text { EHR integration, billing. Dedicated to the practice of gastroenterology }\end{array}$ \\
\hline Cisco Webex meetings ${ }^{\mathrm{TM}}$ & Provides platforms for Webex rooms, web and videoconferencing \\
\hline Doxy.me $\mathrm{TM}^{\mathrm{TM}}$ & $\begin{array}{l}\text { Secure video with the built-in consent process. A free platform that supports a waiting } \\
\text { room workflow }\end{array}$ \\
\hline Carena $^{\mathrm{TM}}$, CarePaths ${ }^{\mathrm{TM}}$, Chrion Health ${ }^{\circledR}$, CloudVisit $^{\mathrm{TM}}$ & $\begin{array}{l}\text { Could be used for virtual platform clinics, behavioral health EHR, secure messaging, } \\
\text { claims, billing, and e-prescribing }\end{array}$ \\
\hline DigiGone $^{\mathrm{TM}}$, Doximity ${ }^{\mathrm{TM}}, 1$ Doc Way ${ }^{\mathrm{TM}}$ & $\begin{array}{l}\text { Subscription model used, DigiGone is used by George Washington University Mari- } \\
\text { time Medical Access to provide telemedicine }\end{array}$ \\
\hline $\mathrm{eVisit}^{\mathrm{TM}}$ & Medical charting, patient emails, onboarding support, 2-way video support \\
\hline GoToMeeting ${ }^{\mathrm{TM}}$ & $\begin{array}{l}\text { Provides video conferencing, desktop sharing via unified communication and collabo- } \\
\text { ration portfolio }\end{array}$ \\
\hline $\begin{array}{l}\text { Secure Telehealth }{ }^{\circledR} \text {, Secure Video }{ }^{\circledR} \text {, Sight Call }{ }^{\mathrm{TM}} \text {, } \\
\text { SnapMD }{ }^{\mathrm{TM}} \text {, Skype for Business/Microsoft meeting }\end{array}$ & $\begin{array}{l}\text { Assists in scheduling, video calling. Secure Video® initially focused on telepsychiatry } \\
\text { but expanded its offering. SightCall uses WebRTC-based video calling platforms }\end{array}$ \\
\hline VSee $^{\mathrm{TM}}$, VirtuMedixm ${ }^{\circledR}$, WeCounsel ${ }^{\mathrm{TM}}$ & $\mathrm{VSee}^{\mathrm{TM}}$ is used by NASA across the international space stations \\
\hline Zoom for Health Care ${ }^{\mathrm{TM}}$ & Offers collaborative healthcare, medical education, and population-based care \\
\hline
\end{tabular}

All platforms need to be compliant with HIPPA rules in order to ensure secure patient and provider communications. Due to the COVID-19 pandemic, HHS temporarily relaxed the restrictions and will not impose penalties for non-compliance provided the care is given with good faith. The list is not complete and is subjected to change based on platform performances and individual vendors. HIPPA—health insurance portability and accountability act, EHR—Electronic health record, NASA—National Aeronautics and Space Administration, ACG-American College of Gastroenterology 


\section{Outpatient GI Referrals and Practice During COVID-19}

With COVID-19, telemedicine has been extended to increase the spectrum of GI encounters to include patients with abdominal pain, colorectal cancer (CRC) screening, abnormal liver tests, and GI bleeding, and liver transplant recipients. In a US study conducted by Serper et al., 94\% of GI/ hepatology visits were virtual via telemedicine during the pandemic compared to only $5 \%$ two weeks before the onset of the COVID-19 [35].

Pre-charting by medical assistants, nurses, and providers (similar to regular in-person visits) can further decrease documentation time and enhance meaningful discussion with the patient [36]. An actively engaging dialogue with the referring physicians' office about the need for additional testing can expedite a definitive treatment plan. A robust EHR with direct patient messaging and available literature can seamlessly engage the communication with referring physicians and the patients providing high-quality care.

\section{Inpatient GI Referrals During COVID-19}

Though telemedicine has been traditionally utilized for dermatology, palliative care, and neurology in the inpatient setting [37, 38], the pandemic has provided a unique opportunity for telemedicine to facilitate timely, high-quality care to patients needing urgent GI care. The CDC added multiple symptoms to the list of COVID-19 extraintestinal manifestations such as nausea, vomiting, dysgeusia, hepatitis, colitis, pancreatitis, and GI bleeding [39, 40]. Moreover, COVID-19 has been associated with abnormal liver chemistries and hyperlipasemia. COVID-19 patients with GI symptoms (including GI bleeding) prompt urgent GI consultations in the inpatient setting. Telemedicine can be utilized in COVID-19 patients with GI symptoms similar to the stroke-related care provided by the neurologists. This need is further increased due to the shortage of COVID-19 testing kits and the need to conserve personnel protective equipment (PPE) while minimizing exposure.

Telemedicine in the inpatient setting could be utilized in multiple ways [30]: inpatient e-consults (involves a precise question with a review of the medical chart and a need for formal written note); inpatient telephonic consults (use of a telephone to obtain relevant information with the review of the chart and documentation of the note, appropriate for questions involving active patient engagement); and inpatient video consults (use of video in an integrated/non-integrated platforms to obtain a full history, limited physical examination with the assistance of bedside nurse, and documentation of full consult note) [41]. Patient care assistants could help set up the necessary equipment and minimize interruptions akin to settings used in acute stroke-related care. A focused examination template for teleconsultations has been proposed in the literature [41]. Regardless of the type of inpatient telemedicine visit, a stable and high-speed Internet connection combined with sensors to measure vital signs and other physical examination aspects is required [18]. Active communication with the referring team can be reimbursed as complex in-person consultations [16].

\section{Use of Telemedicine for Specific GI Disorders During COVID-19 Pandemic}

Though traditionally, telemedicine in GI disorders is restricted to stable, non-urgent problems in outpatient settings, with the advent of the pandemic, this has been extended to urgent and emergent situations as well. Inpatient-type telemedicine can be utilized to provide care for inpatients during the pandemic, especially those who need immediate attention, yet decrease the risk of viral transmission while conserving PPE.

\section{IBD Care}

IBD care with telemedicine has been used for many years. The development of Home Automated Tele management (HAT) was well accepted by IBD patients [42]. Finkelstein et al. [43] reported using HAT in 2003 for chronic diseases such as asthma, chronic obstructive lung disease (COPD) and extended it to IBD care. It was originally developed for patients with different chronic health conditions in order to facilitate their self-care [43]. It involves using a networked computer with an automated transfer of patient survey results to a central server to track disease progression [6]. Multiple institutions have development platforms such as IBD Qorus ${ }^{\mathrm{TM}}$ program centers [44], HealthPROMISE [45], IBD LIVE ${ }^{\mathrm{TM}}$ (Liver Interinstitutional and interdisciplinary Video conference Education) [46], and myIBDcoach [47] in order to provide high-quality care, improve adherence to the treatment regimens, and identify disease progression at early stages facilitating the timely institution of therapies. There is no better time for increased telemedicine utilization in IBD care than now $[48,49]$. Although IBD patients may be at higher risk for acquiring SARS-CoV-2 infection due to a dysregulated immune system, biological therapy continuation in IBD is essential. Telemedicine could be utilized in outpatient and inpatient settings in these patients to provide care with an emphasis on medication adjustments (biological therapy, corticosteroid, and antiinflammatory agent use). Moreover, IBD patients using online applications expressed the desire to increase their 
use during the pandemic [50]. Interestingly fecal calprotectin testing, an integral part of the management of IBD patients, has been reduced during the pandemic due to a theoretical risk of fecal transmission of SARS-CoV-2 and contact with laboratories [50, 51]. Lewin et al. reported that telemedicine use in IBD increased to $50 \%$ or more, compared with $<25 \%$ in the pre-pandemic era [41]. An international survey from 56 countries and 802 respondents reported that $0-25 \%$ of pre-pandemic IBD clinical encounters were telemedicine compared with $>75 \%$ during the pandemic [50]. Furthermore, it is anticipated that $50 \%$ of the clinical encounters will be continued via telemedicine after COVID-19-related restrictions are lifted [50], emphasizing the increasing utility of telemedicine for IBD care, which will likely continue post-pandemic.

\section{Hepatology Care}

Telemedicine has been successfully used for many years in HCV management. For example, in 2003, the University of New Mexico Health Sciences developed a model called "Extension for Community Healthcare Outcomes (ECHO)" to improve access to chronic disease management (such as hepatitis C) in rural areas of New Mexico. This model uses the inter-disciplinary expertise of specialty providers to assist in complex disease management of hepatitis C. Furthermore, co-management of patients with the engagement of PCPs can facilitate interactive case-based discussions and improving outcomes. The Veterans Affairs Health System adopted a similar model (VAHS) called Specialty Access Network-Extension of Community Healthcare Outcome (SCAN-ECHO) to deliver care to patients with liver disease [52]. Most telemedicine encounters in hepatology involve HCV management. Few studies have evaluated telemedicine's utility in chronic liver disease management, hepatocellular carcinoma, peri-procedural/surgical management of patients with advanced liver disease, and determining patients' liver transplant status with decompensated cirrhosis [53]. Due to these changes, "telehepatology" programs have emerged during the COVID-19 pandemic [9]. With the advent of COVID-19, a renewed interest emerged to incorporate telemedicine beyond $\mathrm{HCV}$ into overall hepatology care. Though integrated health systems such as VAHS and Kaiser Permanente have utilized telemedicine in order to reduce cost and increase access to patients in the past [54], smaller systems still have challenges in achieving telemedicine in hepatology care due to multiple barriers (such as integrating the care, limited by the type of electronic health record system, and financial components, Table 1). It is expected that telemedicine will be accepted for a wider range of liver diseases with COVID-19 emergence and relaxation of reimbursement restrictions.

\section{Outpatient Gastroenterology Services}

With telemedicine's success in IBD and hepatology care, its utility has been extended recently with the emergence of COVID-19. Tang and colleagues reported a model for all outpatient gastroenterology referrals over five months, during which 1243 hybrid electronic consultations were performed with an $887(71 \%)$ reduction of the in-person need for a clinic visit [36]. Furthermore, $98 \%$ of consultations were completed within $20 \mathrm{~min}$. These visits were most commonly utilized for CRC screening/surveillance and GI symptoms such as abdominal pain and heartburn. Telemedicine is currently being employed in all non-emergent outpatient GI visits, especially during the pandemic. Functional GI disorders such as irritable bowel syndrome (IBS), chronic abdominal pain, and post-procedure monitoring are all managed via telemedicine [55]. The GI conditions needing further evaluation can be followed by repeated telehealth visits or an in-person visit if needed.

\section{Barriers to Telemedicine}

Legal challenges, reimbursement restrictions, and state licensure requirements [55] had traditionally limited telemedicine's widespread applicability in gastroenterology (Table 1). Moreover, telemedicine has not yet become a part of formal GI training, causing discomfort during visits among novice gastroenterologists. Though these restrictions have been relaxed during the pandemic with the remarkable growth of telehealth, other barriers (mentioned below) persist. These could be divided into the patient and provider-related factors (Fig. 2). Patient factors - traditionally, though inpatient visits start with a handshake, history, and a "hands-on" physical examination that were the core of the patients' visit [56], the use and time spent on the physical examination have been decreasing with the growth of technology, advanced imaging, and laboratory data. Nevertheless, the physical examination is the inherent part of the visit, especially when testing modalities are not available [57]. The COVID-19 pandemic changed this paradigm due to a need to maintain social distancing and stay-at-home orders. Furthermore, older patients can have hearing problems and sometimes rely on lip reading. The lack and the requisite knowledge to navigate and operate necessary electronic equipment are also a major limitation in older patients. It can be challenging to adapt to telehealth changes for some accustomed to traditional methods. Acceptability of telemedicine was notably low among the Black and elderly patients, higher-risk groups for severe COVID-19, for unknown reasons [35] [58]. These barriers can be overcome in part by medical assistants who can act as facilitators to engage the patient in a telephone or televideo visit prior to 
the provider visit, allaying anxiety while providing technical support, helping troubleshoot the potential issues can arise during the video chat with the provider.

Provider factors - Similar to patient factors, providers are familiar with the paradigm of F2F visits, often relying on non-verbal cues to connect with their patients [16], while keeping the patient-provider relationship intact by engaging the patient and the family members accompanying them. A survey of GI providers in April 2020 showed that $68 \%$ of respondents never performed video consultations prior to the pandemic [41]. With the increasing use of EHR, online portal use, and communications with patients via online messaging, the utility of telemedicine has been improving [59]. Although video consultations can assist with nonverbal cues, including facial expressions, telephone visits are technically easier and more flexible. These factors, combined with the need for interstate licensing, easier reimbursement policies, adoption of a similar level of reimbursements for in-person visits, and no cost-sharing for patients, have helped telemedicine expand at a rapid pace. Despite these promising developments, unidentified barriers, risks of privacy loss, a breach in patient-provider relationship, loss of information, hacking, breach of HIPPA, confusion about ordering tests, whether referring provider or specialty provider could occur, which can improve with continuing multidisciplinary efforts in the future.

\section{The Future of Telemedicine in Gastroenterology}

With all the changes noted recently, telemedicine adoption has increased significantly and is expected to rise further. As the COVID-19 pandemic unfolds globally, temporary relaxations of telemedicine usage restriction will only accelerate its widespread use. Although this is unchartered territory due to the ongoing pandemic, lessons learned from telemedicine during this public health crisis will be used for many years. Telemedicine is a lowcost, widely available tool that can reach patients with multiple barriers to in-person visits. If utilized properly, with the relaxation of rules for its widespread use with the appropriate patient and provider education, it can greatly assist in providing patient-centric care with the improved overall quality. Moreover, telemedicine is likely the optimal means to reduce the size of backlogs and delays inclinic appointments so far created by the pandemic. It can prioritize the in-person visits to areas that need complex medical care and detailed discussions. With all the available data, telemedicine is feasible, efficient, and does not compromise the quality and clinical care needed for these patients. The future rests with taking a proactive approach with providing patients with options of the type of visit on which to choose based on their lifestyle and convenience.
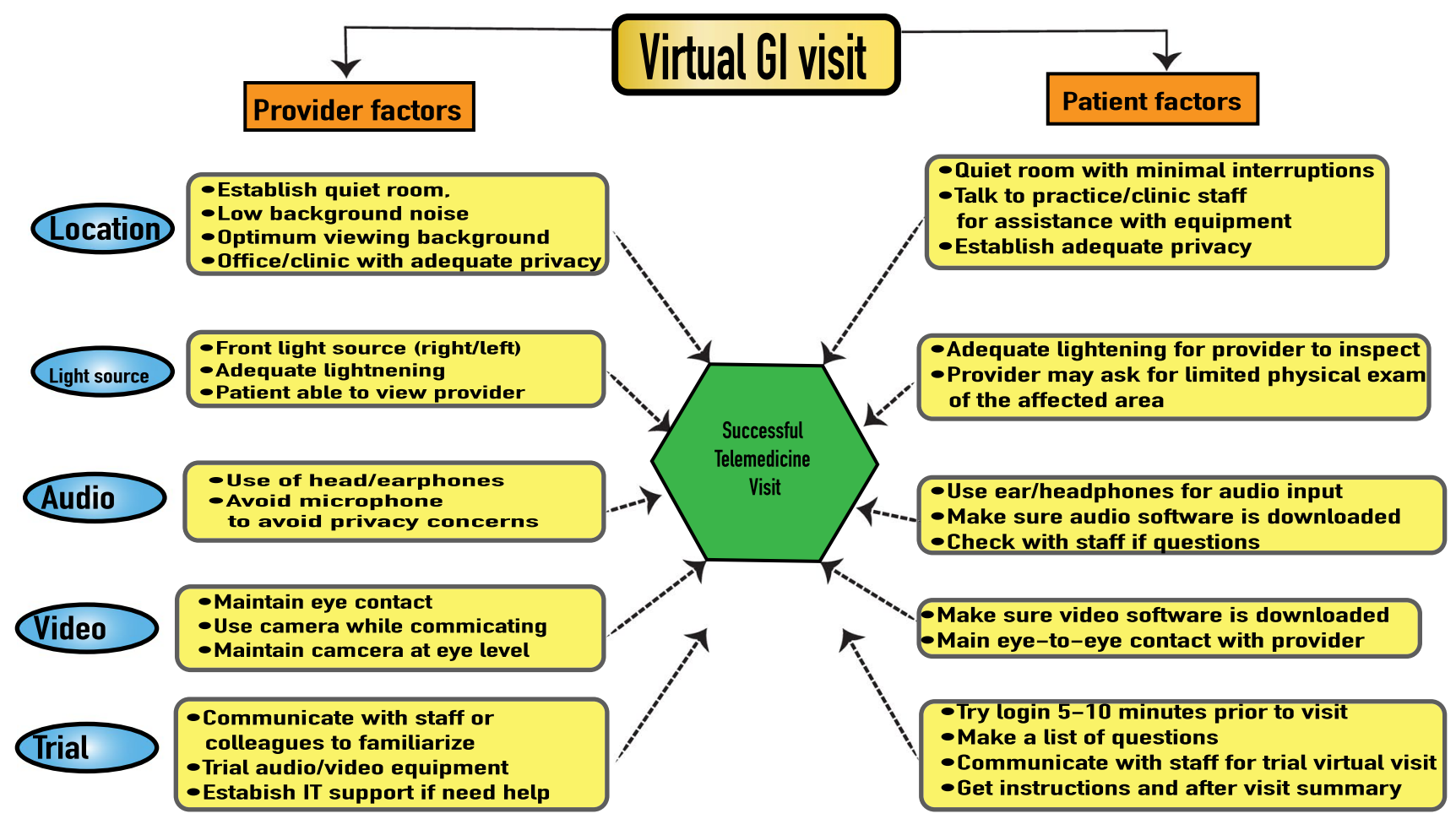

Fig. 2 Patient and provider factors that could favorably modify the telemedicine experience 
Further, it can reduce ED utilization while focusing on high-quality, low-cost care. Outcomes of telemedicine use in the COVID-19 pandemic can be taken as an opportunity by the policymakers and healthcare organizations to develop strategies to improve access and safety in gastroenterology and hepatology specialty care.

Author's contributions HG, AP helped in conception and design, literature review, critical revision and editing, and final approval and AP contributed to first draft.

\section{Compliance with Ethical Standards}

Conflict of interest Hemant Goyal owns stock of Conversion Labs, Inc.

\section{References}

1. Wootton R. Telemedicine. BMJ. 2001;323:557-560

2. Perednia DA, Allen A. Telemedicine technology and clinical applications. JAMA. 1995;273:483-488

3. WHO. TELEMEDICINE Opportunities and developments in Member States. Global Observatory for eHealth series - Volume 2. 2010. https://www.who.int/goe/publications/goe_telemedici ne_2010.pdf. Accessed December 162020.

4. Scurlock C, D'Ambrosio C. Telemedicine in the intensive care unit: state of the art. Crit Care Clin. 2015;31:187-195. https:// doi.org/10.1016/j.ccc.2014.12.001.

5. Gutovitz S, Leggett J, Hart L, Leaman SM, James H, Stillinger $\mathrm{T}$. The impact of tele-neurologists on acute stroke care at an advanced primary stroke centre. $\mathrm{J}$ Telemed Telecare. 2020;13:1357633X20921039.

6. Cross RK, Cheevers N, Rustgi A, Langenberg P, Finkelstein J. Randomized, controlled trial of home telemanagement in patients with ulcerative colitis (UC HAT). Inflamm Bowel Dis. 2012;18:1018-1025

7. Elkjaer M, Shuhaibar M, Burisch J, Bailey Y, Scherfig H, Laugesen B et al. E-health empowers patients with ulcerative colitis: a randomised controlled trial of the web-guided 'Constant-care' approach. Gut. 2010;59:1652-1661

8. Spiegel B. 2015 American Journal of Gastroenterology Lecture: how digital health will transform gastroenterology. Am J Gastroenterol. 2016;111:624-630

9. Serper M, Cubell AW, Deleener ME, Casher TK, Rosenberg DJ, Whitebloom D et al. Telemedicine in liver disease and beyond: can the COVID-19 crisis lead to action? Hepatology. 2020;72:723-728

10. Hollander JE, Carr BG. Virtually perfect? Telemedicine for COVID-19. New England J Med 2020;382:1679-1681

11. Perisetti A, Gajendran M, Boregowda U, Bansal P, Goyal H. COVID-19 and gastrointestinal endoscopies: current insights and emergent strategies. Dig Endosc. 2020;32:715-722. https://doi. org/10.1111/den.13693.

12. Perisetti A, Goyal H, Gajendran M, Boregowda U, Mann R, Sharma N. Prevalence, mechanisms, and implications of gastrointestinal symptoms in COVID-19. Front Med (Lausanne). 2020;7:588711. https://doi.org/10.3389/fmed.2020.588711.

13. Repici A, Maselli R, Colombo M, Gabbiadini R, Spadaccini M, Anderloni A et al. Coronavirus (COVID-19) outbreak: what the department of endoscopy should know. Gastrointest Endosc. 2020;92:192-197. https://doi.org/10.1016/j.gie.2020.03.019.

14. Ward MM, Merchant KA, Carter KD, Zhu X, Ullrich F, Wittrock A, et al (2018) Use of telemedicine for ED physician coverage in critical access hospitals increased after CMS policy clarification. Health Affairs 37:2037-2044.

15. CMS. MEDICARE TELEMEDICINE HEALTH CARE PROVIDER FACT SHEET. 2020. https://www.cms.gov/newsroom/ fact-sheets/medicare-telemedicine-health-care-provider-fact-sheet . Accessed December 52020.

16. Shah ED, Amann ST, Karlitz JJ. The time is now: a guide to sustainable telemedicine during COVID-19 and beyond. Am J Gastroenterol. 2020;115:1371-1375. https://doi.org/10.14309/ ajg.0000000000000767.

17. Rayman R. Telemedicine: military applications. Aviat Space Environ Med. 1992;63:135-137

18. Wilcock AD, Rose S, Busch AB, Huskamp HA, Uscher-Pines L, Landon $\mathrm{B}$ et al. Association between broadband internet availability and telemedicine use. JAMA Intern Med. 2019;179:1580-1582

19. Cross RK, Kane S. Integration of telemedicine into clinical gastroenterology and hepatology practice. Clin Gastroenterol Hepatol. 2017;15:175-181

20. Johnson KD, Harris C, Cain JK, Hummer C, Goyal H, Perisetti A. Pulmonary and extra-pulmonary clinical manifestations of COVID-19. Front Med. 2020;7:526

21. Gadiparthi C, Perisetti A, Sayana H, Tharian B, Inamdar S, Korman A. Gastrointestinal bleeding in patients with severe SARSCoV-2. Am J Gastroenterol. 2020;115:1283-1285

22. Ramachandran P, Onukogu I, Ghanta S, Gajendran M, Perisetti A, Goyal H et al. Gastrointestinal symptoms and outcomes in hospitalized coronavirus disease 2019 patients. Dig Dis. 2020;38:373-379

23. Perisetti A, Gajendran M, Mann R, Elhanafi S, Goyal H COVID-19 extrapulmonary illness - special gastrointestinal and hepatic considerations. Dis Mon. 2020;66:101064. https://doi. org/10.1016/j.disamonth.2020.101064.

24. Aziz M, Perisetti A, Lee-Smith WM, Gajendran M, Bansal P, Goyal H. Taste changes (dysgeusia) in COVID-19: a systematic review and meta-analysis. Gastroenterology. 2020;159:1132-1133

25. Franzini TAP, Kotinda A, Moura DTH, Badana MLV, Medeiros MS, Lima PGR et al. Approach to endoscopic procedures: a routine protocol from a quaternary university referral center exclusively for coronavirus disease 2019 Patients. Clinics (Sao Paulo). 2020;75:e1989. https://doi.org/10.6061/clinics/2020/e1989.

26. Moraveji S, Thaker AM, Muthusamy VR, Banerjee S. Protocols, personal protective equipment use, and psychological/financial stressors in endoscopy units during the COVID-19 pandemic: a large survey of hospital-based and ambulatory endoscopy centers in the United States. Gastroenterology. 2020;159:1568-70.e5. https://doi.org/10.1053/j.gastro.2020.05.061.

27. Sun $\mathrm{S}, \mathrm{Yu} \mathrm{K}, \mathrm{Xie} Z$, Pan X. China empowers Internet hospital to fight against COVID-19. J Infect. 2020;81:e67-e68. https://doi. org/10.1016/j.jinf.2020.03.061.

28. Baker J, Stanley A. Telemedicine technology: a review of services, equipment, and other aspects. Current Allergy Asthma Rep. 2018;18:60

29. Lin JC, Kavousi Y, Sullivan B, Stevens C. Analysis of outpatient telemedicine reimbursement in an integrated healthcare system. Ann Vascular Surgery. 2020;65:100-106

30. Lewin S, Lees C, Regueiro M, Hart A, Mahadevan U. International organization for the study of inflammatory bowel disease: global strategies for telemedicine and inflammatory bowel diseases. J Crohn's Colitis. 2020;14:S780-S4.

31. Telehealth H. Notification of Enforcement Discretion for Telehealth Remote Communications During the COVID-19 Nationwide Public Health Emergency. 2020. https://www.hhs.gov/hipaa 
/for-professionals/special-topics/emergency-preparedness/notif ication-enforcement-discretion-telehealth/index.html. Accessed December 162020.

32. VSee. Telemedicine Platform Reviews. 2020. https://vsee.com/ telemedicine-platform-reviews/. Accessed December 162020.

33. Jnr BA. Use of telemedicine and virtual care for remote treatment in response to COVID-19 pandemic. J Med Syst. 2020;44:1-9

34. Kissani N, Lengané YTM, Patterson V, Mesraoua B, Dawn E, Ozkara $\mathrm{C}$ et al. Telemedicine in epilepsy: how can we improve care, teaching, and awareness? Epilepsy Behav. 2020;103:106854

35. Serper M, Nunes F, Ahmad N, Roberts D, Metz DC, Mehta SJ. Positive early patient and clinician experience with telemedicine in an academic gastroenterology practice during the COVID-19 pandemic. Gastroenterology. 2020;159:1589-91. e4.

36. Tang Z, Dubois S, Soon C, Agrawal D. A model for the pandemic and beyond: Telemedicine for all outpatient gastroenterology referrals reduces unnecessary clinic visits. J Telemed Telecare. 2020;NA:1357633x20957224. doi:https://doi.org/10.1177/13576 $33 \times 20957224$.

37. Worster B, Swartz K. Telemedicine and palliative care: an increasing role in supportive oncology. Curr Oncol Rep. 2017;19:37

38. Hafi NB, Uvais N. Palliative dermatology-an area of care yet to be explored. Prog Palliat Care. 2018;26:187-191

39. Goyal H, Sachdeva S, Perisetti A, Mann R, Inamdar S, Tharian B. Hyperlipasemia and potential pancreatic injury patterns in COVID-19: a marker of severity or innocent bystander? Gastroenterology. 2020;S0016-5085:35326-35329. https://doi. org/10.1053/j.gastro.2020.10.037.

40. Kopel J, Perisetti A, Gajendran M, Boregowda U, Goyal H. Clinical insights into the gastrointestinal manifestations of COVID-19. Dig Dis Sci. 2020;65:1932-1939. https://doi.org/10.1007/s1062 0-020-06362-8.

41. Lewin S, Lees C, Regueiro M, Hart A, Mahadevan U. International organization for the study of inflammatory bowel disease: global strategies for telemedicine and inflammatory bowel diseases. J Crohns Colitis. 2020;14:S780-s4. doi:https://doi. org/10.1093/ecco-jcc/jjaa140.

42. Castro HK, Cross RK, Finkelstein J. Using a Home Automated Telemanagement (HAT) system: experiences and perceptions of patients with inflammatory bowel disease. AMIA Annu Symp Proc. 2006;2006:872

43. Finkelstein J, Khare R, Vora D. Home automated telemanagement (HAT) system to facilitate self-care of patients with chronic diseases. J Syst Cybern Inform 2003;1:78-82

44. Johnson LC, Melmed GY, Nelson EC, Holthoff MM, Weaver SA, Morgan TS et al. Fostering collaboration through creation of an IBD learning health system. Am J Gastroenterol. 2017;112:406408. https://doi.org/10.1038/ajg.2017.9.

45. Atreja A, Khan S, Rogers JD, Otobo E, Patel NP, Ullman T et al. Impact of the mobile HealthPROMISE platform on the quality of care and quality of life in patients with inflammatory bowel disease: study protocol of a pragmatic randomized controlled trial. JMIR Res Protoc. 2015;4:e23. https://doi.org/10.2196/respr ot. 4042 .

46. Regueiro MD, Greer JB, Binion DG, Schraut WH, Goyal A, Keljo $\mathrm{DJ}$ et al. The inflammatory bowel disease live interinstitutional and interdisciplinary videoconference education (IBD LIVE) series. Inflamm Bowel Dis. 2014;20:1687-1695. https://doi. org/10.1097/mib.0000000000000187.

47. de Jong MJ, Boonen A, van der Meulen-de Jong AE, RombergCamps MJ, van Bodegraven AA, Mahmmod N et al. Cost-effectiveness of telemedicine-directed specialized vs standard care for patients with inflammatory bowel diseases in a randomized trial. Clin Gastroenterol Hepatol. 2020;18:1744-1752. https://doi. org/10.1016/j.cgh.2020.04.038.

48. Del Hoyo J, Aguas M. Implementing Telemedicine in Inflammatory Bowel Disease: is COVID-19 the definitive trigger? Gastroenterología y Hepatología (English Edition). 2020;43:415

49. Gajendran M, Perisetti A, Aziz M, Raghavapuram S, Bansal P, Tharian B et al. Inflammatory bowel disease amid the COVID-19 pandemic: impact, management strategies, and lessons learned. Ann Gastroenterol 2020;33:591-602

50. Lees CW, Regueiro M, Mahadevan U. Innovation in inflammatory bowel disease care during the COVID-19 pandemic: results of a global telemedicine survey by the international organization for the study of inflammatory bowel disease. Gastroenterology. 2020;159:805-8.e1. https://doi.org/10.1053/j.gastro.2020.05.063.

51. Perisetti A, Gajendran M, Goyal H. Putative mechanisms of Diarrhea in COVID-19. Clin Gastroenterol Hepatol. 2020;18:30543055. https://doi.org/10.1016/j.cgh.2020.06.008.

52. Su GL, Glass L, Tapper EB, Van T, Waljee AK, Sales AE. Virtual consultations through the veterans administration SCAN-ECHO project improves survival for veterans with liver disease. Hepatology. 2018;68:2317-2324. https://doi.org/10.1002/hep.30074.

53. Serper M, Volk ML. Current and future applications of telemedicine to optimize the delivery of care in chronic liver disease. Clin Gastroenterol Hepatol. 2018;16:157-61.e8. https://doi. org/10.1016/j.cgh.2017.10.004.

54. Portnoy J, Waller M, Elliott T. Telemedicine in the Era of COVID19. J Allergy Clin Immunol Practice. 2020;8:1489-1491

55. Chey WD, Keefer L, Whelan K, Gibson PR. Behavioral and diet therapies in integrated care for patients with irritable bowel syndrome. Gastroenterology. 2020;5085:35281-35291. https://doi. org/10.1053/j.gastro.2020.06.099.

56. Hjelm N. Benefits and drawbacks of telemedicine. J Telemed Telecare. 2005;11:60-70

57. Gustke SS, Balch DC, West VL, Rogers LO. Patient satisfaction with telemedicine. Telemed J. 2000;6:5-13

58. Kopel J, Perisetti A, Roghani A, Aziz M, Gajendran M, Goyal H. Racial and gender-based differences in COVID-19. Front Public Health. 2020;8:418

59. Koulaouzidis G, Marlicz W, Koulaouzidis A. Telemedicine in the time of COVID-19: better late than never. Am J Gastroenterol. 2020;NA(NA):ahead of print. doi:https://doi.org/10.14309 /ajg.0000000000001035.

Publisher's Note Springer Nature remains neutral with regard to jurisdictional claims in published maps and institutional affiliations. 